\title{
The diagnostic accuracy of the mobile phone teledermatoscopy
}

\author{
Hamza Yildiz $^{1^{*}}$, Memet Ersan Bilgili ${ }^{1}$, Hasan Aktug Simsek ${ }^{2}$ \\ ${ }^{1}$ Department of Dermatology, Yunus Emre Government Hospital, Turkey \\ ${ }^{2}$ Department of Pathology, Eskisehir Yunus Emre Government Hospital, Turkey
}

\begin{abstract}
The positive predictive value (PPV) of smart mobile phone teledermatoscopy is not known. The main purpose of the present study was to investigate the sensitivity and positive predictive values (PPVs) of smart mobile phone teledermatoscopy. Over a period of 6 months, up to three clinical and dermatoscopic images were obtained of 67 skin lesions from 67 patients using a mobile phone camera and standard pocket dermatoscopy device. Out of the 67 patients, 44 were men $(65.67 \%)$ and 23 were women $(34.32 \%)$. The mean age of the patients was $39.56 \pm 22.19$ years (ranging from 18 to 92$)$. The majority of the lesions $(71.64 \% ; n=48)$ were benign, while $11.94 \%(n=8)$ of the biopsies were premalignant and $16.41 \%(\mathrm{n}=11)$ of the lesions were malignant. The sensitivity for the diagnosis of benign, premalignant, and malignant lesions were $93.8 \%, 100 \%$, and $100 \%$, respectively. PPVs for the diagnosis of benign, premalignant, and malignant lesions were $93.8 \%, 100 \%$, and $100 \%$, respectively. The sensitivity and PPVs of all lesions were $95.9 \%$ and $95.7 \%$. The accuracy of the teledermatoscopic consultation with a mobile phone is very high. We therefore think that it can be a cost effective and useful method in the consultation at distance.
\end{abstract}

Keywords: skin lesions; smart mobile phone; teledermatology; teledermatoscopy

Citation: Yildiz H, Bilgili ME, Simsek HA, The diagnostic accuracy of the mobile phone teledermatoscopy. J Surg Dermatol 2022; 7(1): 178; http://dx.doi.org/10.18282/jsd.v7.i1.178.

*Correspondence to: Hamza Yildiz, Department of Dermatology, Yunus Emre Government Hospital, Turkey; hamzayildiz@ gmail.com

Received: $23^{\text {rd }}$ September 2021; Accepted: $4^{\text {th }}$ November 2021; Published Online: $24^{\text {th }}$ November 2021

\section{Introduction}

Dermatoscopy ensures better visual image of deeper structures of the skin. Nowadays, it is commonly used and widely accepted screening device in dermatology ${ }^{[1]}$. To overcome the problem of maldistribution of dermatologist, teleconsultation technologies (teledermatology with or without teledermatoscopy) are being used ${ }^{[2]}$. Teledermatoscopy improves the diagnostic accuracy for pigmented or non-pigmented skin lesions ${ }^{[3]}$.

Digital dermatoscopy systems, attached high-end digital cameras and computer are expensive. These complex and expensive techniques may not importantly upgrade management plans and diagnosing. They are also not yet easily approachable. Currently, standard pocket dermatoscopy tools and mobile camera phones are widely distributed, easily available, cheap, reachable, and effective $^{[1,4,5]}$.

In this study, we assessed the sensitivity and positive predictive values (PPVs) of mobile teledermatoscopy (using a mobile camera phone and standard pocket dermatoscopy device).

\section{Methods}

This study was a prospective, open-label, non-randomized controlled clinical study of the diagnostic accuracy of mobile teledermatoscopy. Ethical approval was obtained from Eskisehir Osmangazi University Clinical Research, Ethical Committee (September 26, 2012; protocol no., $2012 / 272$ ) for this study. The study period was from January 2015 to December 2015. The study protocol complied with the ethical guidelines of the Declaration of Helsinki of the World Medical Association.

Patients were selected randomly from the outpatient clinic at the department of dermatology, Eskisehir Military Hospital, Eskisehir, Turkey. Patients with suspicious skin lesion deemed to need a biopsy or excision were included to study.

Copyright $(2022$ Yildiz H, et al. This is an Open Access article distributed under the terms of the Creative Commons Attribution-NonCommercial 4.0 International License (http://creativecommons.org/licenses/by-nc/4.0/), permitting all non-commercial use, distribution, and reproduction in any medium, provided the original work is properly cited. 
Clinical and dermatoscopic figures of each lesion and clinical information were sent to a teledermatologist for decision-making.

\section{Clinical information form}

Clinical information (patient history, sex, age, location and lesion onset etc.) were written in the standard information form for each patient. This form contained clinical information such as age, sex, presenting complaint (does it itch, burn, and hurt?), brief summary of patient's lesion history, localization, onset time (when did it start?), dissemination pattern of the lesion, provocative factor, previous treatment(s), occupation, additional findings, skin types, personal and family history of skin cancer.

\section{Clinical and dermatoscopic images}

Clinical and dermatoscopic images of the lesions were obtained for each lesion (Figure 1). Standard guidelines and previous studies were followed for digital imaging. Two macro images (distance and close-up) at 8.0 megapixel resolutions (4320 X 3240 pixels), 180 dpi (dots per inch), Joint Photographic Experts Group (JPEG) format were obtained for each lesion using a mobile phone (Galaxy Note 4, Samsung). Two dermatoscopic images were also taken for each lesion with the same mobile phone and a lens attachment (Dermlite DL1, 3Gen Inc).

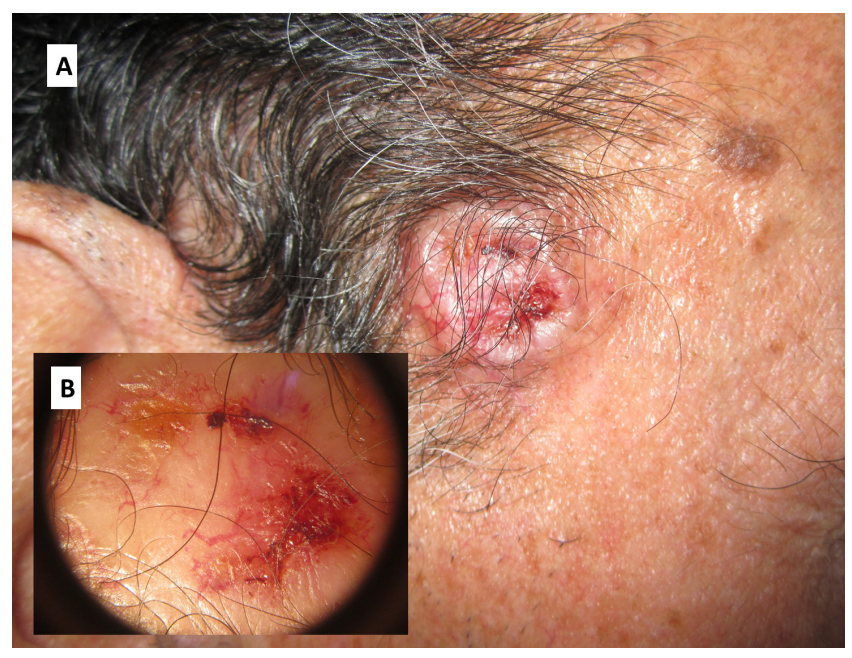

Figure 1. A: A macro image (close-up) of a basal cell carcinoma B. Dermatoscopic image of the same lesion with the same smart mobile phone and a lens attached.

The standard information forms and clinical and dermatoscopic images of the lesions were sent to teledermatologist.

The teledermatologist reported one primary and one differential diagnosis. The results were then compared with a gold standard data to evaluate the teledermatoscopy method. In the present study, not only face-to-face examination but also histopathology was used as a gold standard data. The positive predictive values (PPVs) and sensitivity of the smart mobile phone teledermatoscopy were calculated.

The sensitivity is the probability of the physicians certainly identifying all of the positive diagnosis of a skin lesion. It is described as true positive (TP) / [TP + false negative $(\mathrm{FN})]$. (Table 1$)^{[6]}$.

The PPV is more important than the sensitivity, in clinical setting. It is described as TP / [ $(\mathrm{TP}+$ false positive (FP)] (Table 1). The sensitivity does not foresee the proportion of accuracy of a particular doctor's diagnosis but the PPV determines the proportion of trueness attributed to a medical doctor particular ${ }^{[6]}$.

\section{Statistical analysis}

Data were entered into an Excel spreadsheet and analysed with the statistical Package for SPSS 11.0 statistical software (SPSS Inc., Chicago, IL). The normal distribution of the quantitative data was tested by using the ShapiroWilk test. The Mann-Whitney U test was used for quantitative data without normal distribution. The Chisquare test was used to compare qualitative data. A P value less than 0.05 was assessed statistical significant. The data are represented as the mean values \pm standard deviation (SD).

\section{Results}

Sixty seven patients with 67 skin lesions were enrolled in the study. Data were collected from January 1, 2015, to December 1, 2015. The average age of these participants was $39.56 \pm 22.19$ (between the age of 18-92) years. Of the 67 patients, 23 were women $(34.32 \%)$ and 44 were men $(65.67 \%)$. The average age of the women was $43.60 \pm 2.43$ (between the age of 10-86) years. The average age of the men was $37.45 \pm 2.09$ (between the age of 18-92) years. The average duration of the malignant, premalignant and benign skin lesion were 4.60 \pm 5.61 (ranging from 1 to 20), $4.75 \pm 2.71$ (ranging from 3 to 10 ), and $10 \pm 7.33$ (ranging from 0.25 to 35 ) years, respectively. The average age of all skin lesions was $8.96 \pm 7.21$ (ranging from 0.25 to 35 ) years.

One malignant melanoma (MM), 9 basal cell carcinomas (BCC), 1 squamous cell carcinoma (SCC), 4 keratoacanthomas, 2 actinic keratoses, 2 dysplastic nevi, 5 seborrheic keratoses, 39 nevi and 4 other benign skin lesions (2 dermatofibromas, lentigo simplex, and trichoepithelioma) were included in the study group. The histopathologic diagnoses are shown in the Table 2.

Based on whether the lesions were malignant, premalignant or benign, lesions divided into 3 subgroups. BCCs, SCCs and MMs were deemed malignant lesions. Keratoacanthomas, actinic keratoses and dysplastic nevi were classified as premalignant lesions. Nevi, lentigo simplex, trichoepitheliomas, seborrheic keratoses, and dermatofibromas were deemed benign lesions. The majority of the lesions $(71.64 \% ; n=48)$ were benign, while $11.94 \%$ $(\mathrm{n}=8)$ of the biopsies were premalignant and $16.41 \%(\mathrm{n}=11)$ of the lesions were malignant (Table 2). BCC was the most common malignancy $(13.43 ; n=9)$ in the present study.

Localizations of the lesions are shown in Table 3. The 
Table 1. Diagram demonstrating how the sensitivity, specificity, positive predictive value and negative predictive value are related.

\begin{tabular}{|c|c|c|c|c|}
\hline & & \multicolumn{2}{|c|}{$\begin{array}{c}\text { Condition } \\
\text { (as determined by "Gold standard") } \\
\text { (Face-to-face examination with histopathological } \\
\text { diagnosis) }\end{array}$} & \\
\hline & & Condition Positive & Condition Negative & \\
\hline \multirow{3}{*}{$\begin{array}{l}\text { (Diagnosis of the } \\
\text { teledermatologist) }\end{array}$} & $\begin{array}{l}\text { Test } \\
\text { Outcome } \\
\text { Positive }\end{array}$ & $\begin{array}{l}\text { True Positive } \\
\text { (TP) }\end{array}$ & $\begin{array}{l}\text { False Positive } \\
\text { (FP) }\end{array}$ & $\begin{array}{l}\text { Positive predictive value: } \\
\text { TP / (TP + FP) }\end{array}$ \\
\hline & $\begin{array}{l}\text { Test } \\
\text { Outcome } \\
\text { Negative }\end{array}$ & $\begin{array}{l}\text { False Negative } \\
(\mathrm{FN})\end{array}$ & $\begin{array}{l}\text { True Negative } \\
\text { (TN) }\end{array}$ & $\begin{array}{l}\text { Negative predictive value: } \\
\mathrm{TN} /(\mathrm{FN}+\mathrm{TN})\end{array}$ \\
\hline & & $\begin{array}{l}\text { Sensitivity: } \\
\text { TP / (TP + FN) }\end{array}$ & $\begin{array}{l}\text { Specificity: } \\
\text { TN / (FP + TN })\end{array}$ & \\
\hline
\end{tabular}

Table 2. Histopathologic diagnoses of the lesions are represented.

\begin{tabular}{lll}
\hline Histopathological Diagnoses & $\mathbf{n}$ & $\mathbf{\%}$ \\
\hline Malignant (total) & 11 & 16.41 \\
\hline$>$ Malignant melanoma & 1 & 1.49 \\
\hline$>$ Basal cell carcinoma & 9 & 13.43 \\
\hline$>$ Squamous cell carcinoma & 1 & 1.49 \\
\hline Premalignant (total) & 8 & 11.94 \\
\hline$>$ Keratoacanthoma & 4 & 5.97 \\
\hline$>$ Dysplastic nevus & 2 & 2.98 \\
\hline$>$ Actinic keratosis & 2 & 2.98 \\
\hline Benign (total) & 48 & 71.64 \\
\hline$>$ Nevus (total) & 39 & 58.20 \\
\hline$\bullet$ Intradermal nevus & 28 & 41.79 \\
\hline$\bullet$ Compound nevus & 5 & 7.46 \\
\hline$\bullet$ Junctional nevus & 4 & 5.97 \\
\hline$\bullet$ Blue nevus & 2 & 2.98 \\
\hline$>$ Seborrheic keratosis & 5 & 7.46 \\
\hline$>$ Other benign skin lesions* & 4 & 5.97 \\
\hline Total & 67 & 100 \\
\hline
\end{tabular}

Table 3. Localizations of the lesions are shown.

\begin{tabular}{lllll}
\hline Histopathological Diagnosis & Head and neck & Chest, abdomen, and back & Lower extremity & Upper extremity \\
\hline Malignant melanoma & 1 & - & - & - \\
Basal cell carcinoma & 8 & 1 & - & - \\
Squamous cell carcinoma & 1 & - & - & - \\
Keratoacanthoma & 4 & - & - & - \\
Dysplastic nevus & - & - & 1 & 1 \\
Actinic keratosis & 1 & - & 1 & - \\
Seborrheic keratosis & 4 & - & - & 1 \\
Nevus & 26 & 11 & 1 & 1 \\
Other skin lesions & 2 & - & 1 & 1 \\
\hline
\end{tabular}


PPVs and the sensitivity values for benign premalignant, and malignant skin lesions are shown in Table 4.

Table 4. The sensitivity values and the PPVs for malignant, premalignant, benign skin lesions and all lesions are represented.

\begin{tabular}{lll}
\hline & Sensitivity (\%) & PPV (\%) \\
\hline$\checkmark$ Malignant & 100 & 91.6 \\
$\checkmark$ Premalignant & 100 & 80 \\
$\checkmark$ Benign & 93.8 & 100 \\
$\checkmark$ All lesions & 95.5 & 95.7 \\
\hline
\end{tabular}

\section{Discussion}

TD has been successfully used for remote diagnosis and consultation ${ }^{[7,8]}$. Moreno-Ramirez et al..$^{\left[{ }^{[9]}\right.}$ have reported that store-and-forward TD is an effective, accurate, reliable and valid approach for routine management of patient referrals dermatology clinics. Dermatoscopy is the most widely accepted and most frequently used screening tool in dermatology as it allows better visualization of deeper structures of the $\operatorname{skin}^{[1]}$. Previous studies have demonstrated that dermatoscopy improves the diagnostic accuracy for pigmented melanocytic and non- melanocytic skin lesions $^{[3]}$.

The ability to diagnose and assess benign skin lesions accurately and to distinguish them from malignant skin lesion is vital. Perednia reported that primary care physicians had uncertainty regarding management of more than one in three patients with dermatological lesions. Perednia assessed that it is notable that just one-tenth of these patients was sent the referra ${ }^{[1,10]}$. TD is a very important method because it is shortening the waiting intervals to the surgical treatment, avoiding unnecessary visit to the hospital, and overcoming the some other problems such as geographic maldistribution and lack of dermatologist ${ }^{[1]}$.

We believe that TD methods should rely on low-cost, simple and high-sensitivity diagnostic procedures. Digital dermatoscopy systems, attached high-end digital cameras and computer are expensive. They are also not yet easily approachable ${ }^{[1]}$. Currently, standard pocket dermatoscopy tools and mobile camera phones are widely distributed, easily available, cheap, reachable, and effective ${ }^{[1,4]}$.

Senel et al. investigated the contribution to the management and reliability of the diagnosis of nonmelanocytic skin tumors (150 patients). They found that the reliability (kappa) enhanced dramatically when dermatoscopy was added $(\mathrm{p}<0.05)$. The accuracy of diagnosis was dramatically enhanced by the additional of dermatoscopic figures, from $85 \%$ to $94 \%$ for dermatologist $\mathrm{A}$ and from $88 \%$ to $95 \%$ for dermatologist $\mathrm{B}^{[11]}$. Kromer et al. assessed 113 skin tumours using mobile phone camera. They compared mobile teledermatoscopy with histopathologic results. The both groups showed equally high sensitivity. The sensitivity of benign nonmelanocytic, benign melanocytic, malign non-melanocytic, and malignant melanocytic lesions were $76 \%, 87 \%$, $97 \%$, and $100 \%$, respectively. They reported clinical and dermoscopic tele-evaluations and reported that clinical image tele-evaluation might be the method of choice for mobile tumour screening (kappa, 0.84) ${ }^{[1]}$. Wu et al. used smart mobile phone in 29 patients with atypical nevi. The diagnostic concordance was 0.87 (Kappa). They suggested that mobile eledermatoscopy is feasible and effective as a method for short-term monitoring of clinically atypical nevi ${ }^{[3]}$. Alexander et al. investigated the accuracy of TD (dermatologist $1 ; 50.7$, dermatologist $2 ; 60.9 \%$ ) and face-toface $(66.7 \%)$ dermatological examinations. They assessed that mobile teledermatoscopy solution may be useful as a triage tool[ ${ }^{[4]}$.

The negative predictive value (NPV) is the rate of patients with negative test results who are truly diagnosed. The PPV is the rate of patients with positive test outcomes who are truly diagnosed ${ }^{[12]}$. Guggenmoos-Holzmann et al. and Har-Shai et al. recently stated that the PPV is the suitable and objective illustrator of clinical diagnoses. The PPV is more patient-focused and is often more relevant to patient care ${ }^{[13,14]}$. The PPVs and sensitivity values the for benign skin lesions $(100 \%, 93.8 \%)$, premalignant $(80 \%$, $100 \%)$, and malignant $(91.6 \%, 100 \%)$ were very high in the present study.

These studies suggested that the diagnostic accuracy proportions of teledermatoscopy with mobile phone were high. It can be used by primary physicians in daily practice.

Our study has some limitations. Changes in the staff may have affected the pathological diagnosis though the pathological assessments were performed by experienced pathologists in this study. Our pathologist was a dermatopathologist. The small number of lesions was another limitation of the study. We enrolled only the patients with suspicious skin lesion deemed to need a biopsy or excision.

In conclusion, the accuracy of the teledermatoscopic consultation with a mobile phone is very high. We therefore think that it can be a cost effective and useful method in the consultation at distance.

\section{Conflict of interest}

The authors declare that there is no conflict of interest.

\section{Funding}

This research received no specific grant from any funding agency in the public, commercial, or not-for-profit sectors.

\section{References}

1. Kromer S, Frühauf J, Campbell TM, Massone C, Schwantzer $\mathrm{G}$, et al. Mobile teledermatology for skin tumour screening: Diagnostic accuracy of clinical and dermoscopic image teleevaluation using cellular phones. Br J Dermatol 2011; 164(5): 973-979. doi: 10.1111/j.1365-2133.2011.10208.x.

2. Dekio I, Hanada E, Chinuki Y, Akaki T, Kitani M, et al. Usefulness and economic evaluation of ADSL-based 
live interactive teledermatology in areas with shortage of dermatologists. Int J Dermatol 2010; 49(11): 1272-1275. doi: 10.1111/j.1365-4632.2010.04572.x.

3. Rosendahl C, Tschandl P, Cameron A, Kittler H. Diagnostic accuracy of dermatoscopy for melanocytic and nonmelanocytic pigmented lesions. J Am Acad Dermatol 2011; 64(6): 1068-1073. doi: 10.1016/j.jaad.2010.03.039.

4. Börve A, Terstappen K, Sanberg C, Paoli J. Mobile teledermoscopy-there's an app for that! Dermatol Pract Concept 2013; 3(2): 41-48. doi: 10.5826/dpc.0302a05.

5. Massone C, Hofmann-Wellenhof R, Ahlgrimm-Siess V, Gabler G, Ebner C, Soyer HP. Melanoma screening with cellular phones. PloS One 2007; 2(5): e483. doi: 10.1371/ journal.pone.0000483.

6. Bilgili ME, Yildiz H, Cengiz BP, Saydam IM. Effect of preoperative evaluation by a dermatologist on diagnostic accuracy. Dermatol Surg 2014; 40(12): 1402-1408. doi: 10.1097/DSS.0000000000000168.

7. Wu X, Oliverias SA, Yagerman S, Chen L, DeFazio J, et al. Feasibility and efficacy of patients-initiated mobile teledermoscopy for short-tern monitoring of clinically atypical nevi. JAVA Dermatol 2015; 151(5): 489-496. doi: 10.1001/ jamadermatol.2014.3837.

8. Yildiz H, Abuaf OK, Bilgili ME. The use of teledermatology in daily practices among dermatologist in Turkey. Turk J
Dermatol 2014; 8: 7-11. doi: 10.4274/tdd.1526.

9. Moreno-Ramirez D, Ferrandiz L, Ruiz-de-Casas A, NietoGarcia A, Moreno-Alvarez P, et al. Economic evaluation of a store-and-forward teledermatology system for skin cancer patients. J Telemed Telecare 2009; 15(1): 40-45. doi: 10.1258/ jtt.2008.080901.

10. Prednia DA, Allen A. Telemedicine technology and clinical applications. JAMA 1995; 133: 171-174. doi:10.1001/ jama.1995.03520300057037.

11. Şenel E, Baba M, Durdu M. The contribution of teledermatoscopy to the diagnosis and management of nonmelanocytic skin tumours. J Telemed Telecare 2013; 19(1): 60-63. doi: 10.1177/1357633X12474961.

12. Altman DG, Bland JM. Diagnostic tests 2: Predictive values. BMJ 1994; 309(6947): 102. doi: 10.1136/bmj.309.6947.102.

13. Har-Shai Y, Hai N, Taran A, Mayblum S, Barak A, et al. Sensitivity and positive predictive values of presurgical diagnosis of excised benign and malignant skin tumors: A prospective study of 835 lesions in 778 patients. Plast Reconstr Surg 2001; 108(7): 1982-1989. doi: 10.1097/00006534200112000-00022.

14. Guggenmoos-Holzmann I, van Houwelingen HC. The (in) validity of sensitivity and specificity. Stat Med 2000; 19: 17831792. doi: 10.1002/1097-0258(20000715)19:13<1783::AIDSIM497>3.0.CO;2-B 